\title{
Prevalence of anxiety and associated factors among people living with HIV/AIDS at Debretabor general hospital Anti Retro Viral clinic Debretabor, Amhara, Ethiopia, 2014
}

\author{
Amsalu Belete ${ }^{1, *}$, Gashaw Andaregie ${ }^{2}$, Minale Tareke ${ }^{3}$, Tigabu Birhan ${ }^{4}$, Telake Azale ${ }^{5}$ \\ ${ }^{1}$ Debretabor general hospital, Debretabor, Ethiopia \\ ${ }^{2}$ Institute of Public health, College of Medicine and Health Science, University of Gondar, Gondar, Ethiopia \\ ${ }^{3}$ Department of Psychiatry, Bahirdar University, Bahirdar, Ethiopia \\ ${ }^{4}$ Department of Medical Laboratory, Debretabor Health Science College, Debretabor, Ethiopia \\ ${ }^{5}$ Institute of Public health, College of Medicine and Health Science, University of Gondar, Gondar, Ethiopia
}

Email address:

amsalu04@gmail.com (A. Belete),tigabu456@gmail.com (T. Birhan)

\section{To cite this article:}

Amsalu Belete, Gashaw Andaregie, Minale Tareke, Tigabu Birhan, Telake Azale. Prevalence of Anxiety and Associated Factors among People Living with HIV/AIDS at Debretabor General Hospital Anti Retro Viral Clinic Debretabor, Amhara, Ethiopia, 2014. American Journal of Psychiatry and Neuroscience. Vol. 2, No. 6, 2014, pp. 109-114. doi: 10.11648/j.ajpn.20140206.15

\begin{abstract}
Introduction: Anxiety is one of the co-morbidities that are often overlooked in treating patients for Acquired Immune Deficiency Syndrome from Human Immunodeficiency Virus (HIV/AIDS). Anxiety is higher among HIV/AIDS than the general population. Anxiety among those that have recently been diagnosed with, HIV has been shown to be more prevalent among patients with stress or excess social stigma related to their diagnosis. Specific prevalence of Anxiety is difficult to identify as a result of the wide variations across the globe, ranging from $7 \%$ to 82.3 OBJECTIVE: The aim of this study was to assess the prevalence of anxiety and associated factors among PLWHA at Debretabor general hospital ART clinic Debretabor,South Gondar, Amhara, Ethiopia, 2014. METHOD: Institute based cross -sectional study was conducted from April 28 to May 28,2014 at Debretabor general hospital among PLWHA. Systematic random sampling method was used; logistic regression was performed to assess the association between binary outcomes and Different explanatory variables and the strength of association was interpreted using odds ratio and confidence interval, P-value $<0.05$ was considered statistically significant in this study. Result: A total of 436 PLWH patients were included in this cross-sectional study. Out of which, 22.2\% of the study participants had anxiety. Respondents who are Being female (AOR $=2.63,95 \%$ CI 1.399, 4.931), those who divorced (AOR $=2.42,95 \%$ CI 1.165 , 5.023), started ART (AOR =2.74, 95\% CI 1.425, 5.264) and perceived stigma (AOR=9.34, 95\% CI 5.435, 16.176) were found to be significantly associated with anxiety. Conclusion and Recommendation: Prevalence of anxiety was found to be high on people living with HIV at Debretabor general hospital ART clinic. Being female, perceived stigma, started ART and divorced were significantly associated to anxiety. These findings highlight the importance of screening and treatment of anxiety as an integral component of HIV care.
\end{abstract}

Keywords: Anxiety, PLWHA, Ethiopia

\section{Introduction}

\subsection{Statement of the Problem}

Anxiety is a vague, subjective, non-specific feeling of uneasiness, apprehension, tension, (excessive nervousness) fears, and a sense of impending doom, irrational avoidance of objects or situation and anxiety attack [1].

Anxiety is one of the co-morbidities that are often overlooked in treating patients for Acquired Immune
Deficiency Syndrome from Human Immunodeficiency Virus (HIV/AIDS), anxiety is higher among HIV/AIDS than the general population. Anxiety among those that have recently been diagnosed with, HIV has been shown to be more prevalent among patients with stress or excess social stigma related to their diagnosis [2].

Globally 33 million people were living with HIV and 2 million people died from AIDS related causes worldwide, particular in sub-Saharan Africa 22 million people living with HIV and 1.2 million people died from AIDS[3].It is estimated 
that 760,000 adults aged 15 to 49 and 380,000 children aged 0 to 14 living with HIV. In Ethiopia170, 000 people deaths due to AIDS [4].

HIV/AIDS imposes a significant psychological burden. People with HIV often suffer from anxiety as they adjust to the impact of the diagnosis of being infected and face the difficulties of living with a chronic life-threatening illness, for instance shortened life expectancy, complicated therapeutic regimens, stigmatization, and loss of social support, family or friends. HIV infection can be associated with high risk of suicide or attempted suicide [5].

People affected by HIV/AIDS are more prone to developing mental disorders such as anxiety, which, in turn, impair their immune function, reduce their quality of life and adherence to treatment and contribute significantly to their premature deaths [6].

Anxiety Disorders is one of the most prevalent mental disorders in the general population, with women affected nearly twice as frequently as men [1]. Anxiety can also correlate with lower adherence to antiretroviral therapy (ART), medical recommendations reduced quality of life, social functioning and excess disability [2].

Specific prevalence of Anxiety is difficult to identify as a result of the wide variations across the globe, ranging from $7 \%$ to $82.3 \%[7,2]$.

\section{Objectives}

\subsection{General Objective}

To assess prevalence of anxiety and associated factors among PLWHA attending ART clinic at Debretabor general hospital, South Gondar, Amhara, Ethiopia, 2014.

\subsection{Specific Objectives}

- To determine prevalence of anxiety among PLWHA attending ART clinic at Debretabor general hospital, South Gondar,Amhara, Ethiopia, 2014

- To identify factors associated with anxiety among PLWHA attending ART clinic at Debretabor general hospital, South Gondar,Amhara, Ethiopia, 2014

\section{Methods}

\subsection{Study Design and Study Period}

Institution based cross-sectional study was conducted from April 28 to May 28, 2014.

\subsection{Study Area}

The study was conducted at Debretabor general hospital, Debretabor town, south Gondar, Ethiopia which is located 103 $\mathrm{km}$ from Bahirdar and $666 \mathrm{~km}$ from the capital city of Ethiopia, Addis Ababa. It is one of the 19 hospital in Amhara region and the only Hospital in South Gondar. The hospital serves approximately 900 PLWHA per month.

\subsection{Source and Study Population}

\subsubsection{Source Population}

All HIV infected patients who were attending ART clinic at Debretabor general hospital.

\subsubsection{Study Population}

HIV infected patients who came to ART clinic at Debretabor general hospital during the study period

\subsection{Sample size determination}

Single population proportion formula were used to calculate the sample size

$$
n=\frac{Z_{\frac{\alpha}{2}}^{2} p(1-P)}{d^{2}}
$$

Where,

$\mathrm{P}=$ by taking the prevalence of anxiety among PLWHA in the same institution based cross-sectional study done in Kenya that was $22.75 \%(22)$.

$\mathrm{D}=$ is the margin of error $(0.04)$, and

$\mathrm{Z} \alpha / 2=1.96$ corresponding to $95 \%$ confidence level.

The resulting sample size becomes 422 participants. Considering $5 \%$ (20) of non response rate will give the total sample size 443 .

\subsubsection{Sampling Procedures}

Systematic random sampling method was used to select patients coming to Debretabor general hospital during the study period from 900 patients. The sample size was decided to be 443 .

The sampling fraction was 900/443=2 hence, the sample interval was 2.The first individual was selected by lottery method and individuals were chosen at regular intervals $\left(\right.$ every $\left.2^{\text {nd }}\right)$ and the selected patients were interviewed by data collectors.

\subsection{Operational Definition}

Anxiety= Individual who scored $\geq 22$ on Beck Anxiety Inventory scale

Perceived sigma $=$ Individual who scored $\geq 1$ on three items Perceived sigma scale

Good Social support $=$ Individual who scored $\geq 9$ on Oslo social support scale.

Ever use of Substance: using of a specific substance at least once in life time.

Current Substance use: using of a specific substance in the last three months.

Data was collected by using a structured questionnaire regarding socio -demographic characteristics and medical data related to HIV/AIDS. Concerning prevalence of anxiety, social support and stigma, Beck Anxiety Inventory scale, Oslo social support scale and stigma scale were used respectively and face to face interview was employed to obtain the data. Beck Anxiety Inventory scale was used to measure the anxiety level of HIV/AIDS patients, Social support was collected by Oslo 3-item social support scale, Oslo 3-item social support 
scale is 3-item questionnaire commonly used to asses' social support and it has been used in several studies, the sum score scale ranging from 3-14, which has three broad categories: "poor support" 3-8, "moderate support" 9-11 and "strong support" 12 and above.(23).

\subsection{Data Processing and Analysis}

The coded data and checked was cleaned by entered into SPSS window version16 and analyzed. Frequencies, percentage, median and chart were used to describe study results. Logistic regression was performed to assess the association between binary outcomes and different explanatory variables. Bivariate analysis was first conducted for each potentially explanatory risk factor. Variables that satisfied P-value $<0.2$ were selected for further analysis using multiple logistic regression models. The strength of association was interpreted using odds ratio and confidence interval. P-value $<0.05$ was considered statistically significant in this study.

\subsection{Ethical Considerations}

Ethical clearance was obtained from the Institutional Review Board (IRB) of college of medicine and health sciences, University of Gondar and Amanuel Mental Specialized Hospital. The data collectors were clearly explain the aims of the study for study participants. Information was collected after write consent and obtained from each participant. The right was given to the study participants to refuse or discontinue participation at any time they want and the chance to ask any thing about the study. Participants were also informed that there is no expectation of additional treatment or any benefits for them associated with participating in the study but that those who scored high on Beck Anxiety Inventory was referred to a specific person at the hospital for assistance. For the purpose of anonymity participant's name wasn't used at the time of data collection and all other personal information kept entirely anonymous and confidentiality was assured throughout the study period. Written consent was obtained from each participant before the start of data collection.

\section{Result}

Socio-demographic characteristics of the respondents

From a total of 443 sample size, 436 were interviewed with response rate of $98.4 \%$. From them $262(60.1 \%)$ were females; $182(41.7 \%)$ lie in the age group of $28-37$ years where as the median age were 37 years which ranges from 20-65years.

Majority of the study participants were married 133 $(30.5 \%)$, orthodox followers $430(98.6 \%)$, merchants 109 (25\%) followed by daily laborers $105(24.1 \%)$, Amhara 436 $(100 \%)$ by ethnicity and regarding educational status 165 $(37.8 \%)$ not educated followed by primary school 133 $(30.5 \%)$

Table 1. Distribution of Socio-demographic characteristics of the prevalence of anxiety among PLWHA at Debretabor general hospital ART clinic, South Gondar, Amhara, Ethiopia, 2014

\begin{tabular}{|c|c|c|c|}
\hline Socio-demographic variables & Category & Frequency & Percent $(\%)$ \\
\hline \multirow{4}{*}{ Age } & $18-27$ & 46 & 10.6 \\
\hline & $28-37$ & 182 & 41.7 \\
\hline & $38-47$ & 129 & 29.6 \\
\hline & $>47$ & 79 & 18.1 \\
\hline \multirow{2}{*}{ Sex } & Male & 174 & 39.9 \\
\hline & Female & 262 & 60.1 \\
\hline \multirow[t]{2}{*}{ Ethnicity } & Amhara & 436 & 100.0 \\
\hline & Married & 133 & 30.5 \\
\hline \multirow{3}{*}{ Marital status } & Single & 109 & 25.0 \\
\hline & Divorce & 118 & 27.1 \\
\hline & Widowed & 76 & 17.4 \\
\hline \multirow{3}{*}{ Religion } & Orthodox & 430 & 98.6 \\
\hline & Muslim & 6 & 1.4 \\
\hline & Not educated & 165 & 37.8 \\
\hline \multirow{3}{*}{ Educational level } & Primary & 133 & 30.5 \\
\hline & Secondary & 73 & 16.7 \\
\hline & More than secondary & 65 & 14.9 \\
\hline \multirow{5}{*}{ Occupation } & Farmer & 97 & 22.2 \\
\hline & Daily labourer & 105 & 24.1 \\
\hline & Governmental employee & 102 & 23.4 \\
\hline & Merchants & 109 & 25.0 \\
\hline & Private employee & 23 & 5.3 \\
\hline \multirow{2}{*}{ Income } & $<712.5$ Ethiopian Birr & 288 & 66.1 \\
\hline & $>=712.5$ Ethiopian Birr & 148 & 33.9 \\
\hline
\end{tabular}

\section{Clinical characteristics of the respondents}

Majority of the respondents $72.2 \%$ were on ART. Almost half of the respondents which is $56.2 \%$ had CD4 counts less than or equal to 500 cells $/ \mu \mathrm{L}$. According HIV/AIDS stage classification Stage I (33.7\%) followed by stage II (28.4\%) and $33.9 \%$ report perceived stigmatized by other people because of their illness and $37.2 \%$ had poor social support.

Based on the cut of point $\geq 22$ for case on Beck Anxiety Inventory the prevalence of anxiety among PLWHA at Debretabor general hospital ART clinic was found to be 
$22.2 \%$.

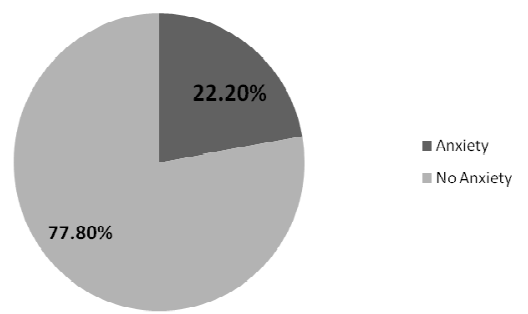

Fig 1. Prevalence of anxiety among PLWHA at Debretabor general hospital ART clinic, South Gondar, Amhara, Ethiopia, 2014

Association of Socio- Demographic and Clinical variables with Anxiety

On bivariate analysis the factors found to fulfill the minimum requirements ( $\mathrm{P}$-value $<0.2$ in this study) were age, sex, income, marital status, educational level ,CD4 cell counts, HIV/AIDS stages, Started taking ART, perceived stigma and social support these factors were entered into multivariate logistic regression for further analysis in order to control confounding effects. However; significant association was not observed between anxiety and study variables like ethnicity, religion, occupation, co morbid disease, both ever and current use of substance at minimum requirement were excluded from further analysis.

The result of multivariate analysis showed that anxiety was significantly associated with sex, marital status, started taking ART and perceived stigma. Sex was significantly associated with anxiety. Being female was 2.6 times (AOR $=2.63,95 \%$ CI $1.399,4.931)$ more likely to have anxiety as compared to male.

Marital status was another factor associated with anxiety. Those who divorced were 2.4 times $(\mathrm{AOR}=2.42,95 \% \mathrm{CI}$ $1.165,5.023)$ more likely to have anxiety as compared to who had married.

Patients who started ART were 2.7 times (AOR $=2.74,95 \%$ CI $1.425,5.264)$ more likely to have anxiety as compared to Patients who didn't start ART.

Moreover patients who report perceived stigma were 9 times more likely to have anxiety as compared to patients who didn't report perceived stigma $(\mathrm{AOR}=9.34,95 \% \mathrm{CI}, 5.435$, 16.176).

On the other hand age, income, educational level, CD4 cell counts, HIV/AIDS stages and social support had no statistically significant association with anxiety on multivariate logistic regression

Table 2. bivariate and multivariate analysis of clinical variables associated with the prevalence of anxiety among PLWHA at Debretabor general hospital ART clinic, South Gondar, Amhara, Ethiopia, 2014

\begin{tabular}{|c|c|c|c|c|c|}
\hline \multirow{2}{*}{ Variable } & \multirow{2}{*}{ Category } & \multicolumn{2}{|c|}{ Anxiety } & \multirow{2}{*}{$\operatorname{COR}(95 \% \mathrm{CI})$} & \multirow{2}{*}{$\operatorname{AOR}(95 \% \mathrm{CI})$} \\
\hline & & Yes & No & & \\
\hline \multirow{4}{*}{ Age } & $18-27$ & 7 & 39 & $.77(.287,2.044)$ & $.369(.112,1.209)$ \\
\hline & $28-37$ & 50 & 132 & $1.61(.844,3.095)$ & $1.03(.469,2.262)$ \\
\hline & $38-47$ & 25 & 104 & $1.03(.503,2.090)$ & $.82(.351,1.912)$ \\
\hline & $>47$ & 15 & 64 & 1 & 1 \\
\hline \multirow{3}{*}{ Sex } & Male & 21 & 153 & 1 & 1 \\
\hline & Female & 76 & 186 & $2.98(1.755,5.050)$ & $2.63(1.399,4.931)^{*}$ \\
\hline & Married & 19 & 114 & 1 & 1 \\
\hline \multirow{3}{*}{ Marital status } & Single & 16 & 93 & $1.03(.503,2.119)$ & $.93(.413,2.067)$ \\
\hline & Divorced & 40 & 78 & $3.08(1.660,5.705)$ & $2.42(1.165,5.023)^{*}$ \\
\hline & Widowed & 22 & 54 & $2.44(1.221,4.893)$ & $1.64(.722,3.741)$ \\
\hline \multirow{4}{*}{ Educational level } & Not educated & 46 & 119 & $2.41(1.101,5.256)$ & $1.48(.589,3.701)$ \\
\hline & Primary & 28 & 105 & $1.66(.732,3.760)$ & $1.49(.580,3.828)$ \\
\hline & Secondary & 14 & 59 & $1.48(.592 .3 .682)$ & $1.48(.509,4.277)$ \\
\hline & More than secondary & 9 & 56 & 1 & 1 \\
\hline \multirow{2}{*}{ Income } & $<712.5$ & 73 & 215 & $1.75(1.052,2.925)$ & $1.04(.515,2.095)$ \\
\hline & $>=712.5$ & 24 & 124 & 1 & 1 \\
\hline \multirow{2}{*}{ CD4 counts } & $=<500$ cells $/ \mu \mathrm{L}$ & 63 & 182 & $1.60(1.000,2.554$ & $1.21(.594,2.456)$ \\
\hline & $>500$ cells $/ \mu \mathrm{L}$ & 34 & 157 & 1 & 1 \\
\hline \multirow{4}{*}{ HIV/AIDS stages } & Stage I & 36 & 111 & 1 & 1 \\
\hline & Stage II & 19 & 105 & $.56(.301,1.034)$ & $.77(.371,1.601)$ \\
\hline & Stage III & 31 & 84 & $1.14(.652,1.987)$ & $.898(.428,1.884)$ \\
\hline & Stage IV & 11 & 39 & $.87(.404,1.874)$ & $.398(.144,1.104)$ \\
\hline \multirow{2}{*}{ Started taking ART } & No & 17 & 104 & 1 & 1 \\
\hline & Yes & 80 & 235 & $2.08(1.175,3.690$ & $2.74(1.425,5.264)^{*}$ \\
\hline \multirow{2}{*}{ Perceived stigma } & No & 27 & 261 & 1 & 1 \\
\hline & Yes & 70 & 78 & $8.68(5.204,14.461)$ & $9.38(5.435,16.176)^{*}$ \\
\hline \multirow{2}{*}{ Social support } & No & 71 & 91 & 7.44(4.471,12.387) & $.543(.062,4.758)$ \\
\hline & Yes & 26 & 248 & 1 & 1 \\
\hline
\end{tabular}

Note: $*=P$ value $\leq 0.05$

$\mathrm{P}$-value of Hosmer and Lemeshew test $=0.659$ 


\section{Discussion}

In the current study, the result showed that the prevalence of anxiety was $22.2 \%$ and this study was similar with the study conducted in Kenya $22.75 \%$ (22). The finding of this study also similar with Nigeria $21.7 \%$ (19).

However; in the present study the prevalence of anxiety was higher the study done in Tanzanian $15.5 \%$ (21), Nigeria $15.6 \%$ (18), Brazil 12.6\% (13), Singapore17.9\% (11) and Journal of the Association of Nurses in AIDS Care reports 7\% (7) The possible reason might be different instrument and socio-cultural difference

The finding of this result was lower than the result reported from India which showed that the prevalence anxiety among people living with HIV/ADIS were 44\%, 29.31\%, 36\% (15, $16,17)$. Also the current result was lower than the result reported from China $45.6 \%$ (14), Brazil 35.8\% (12), Albania $82.3 \%$ (2) Italy 47\%, (10) and USA 33\%, 35.5\% (8, 9) respectively which indicated that the prevalence of anxiety among people living with HIV/ADIS. The possible reason for the different might be different instrument and socio-cultural difference.

Regarding associated factors, in this study, being females were to have anxiety as compared to males which was similar with the study done in Barzile,India, china respectively $(13,14$, and 16). The possible reason might be increased exposure to acute life events, chronic social stresses, lower social status, lower income, and smaller social networks.

When anxiety was in relation to divorce, those who divorced were more likely to have anxiety as compared to who had married. One of the effects of divorce was it destroys the whole dynamic of the family. The possible reason might be due to the stress of divorce, current financial hardship and lack of confidence.

The result of the present study also revealed that the prevalence of anxiety was significantly influenced by perceived stigma about their HIV status which was consistent with study done in Albania (2). One of the most distressing factors of individuals with HIV infection is stigma. The possibility of stigmatizing situation serving as the event of provoking anxiety. The possible reason might be Illiteracy and lack of knowledge regarding the nature of transmission and possible treatment of HIV infection contributes to the high level of stigma and low self esteem.

Patients who started ART were 2.7 times more likely to have anxiety as compared to Patients who didn't start ART. This finding was consistent with in other studies $(2,7)$. The possible reason might be anti retroviral drugs used in treatment of HIV/AIDS to cause anxiety as possible side effect especially efavirenz and nevirapine. Once started, antiretroviral treatment must be taken every day for life. Every missed dose increases the risk that the drugs will stop working this may lead fear of dying. Patients' CD4 count is an important factor in the decision to start ART. A low or falling CD4 count indicates that HIV is advancing and damaging the immune system .A rapidly decreasing CD4 count increases the urgency to start ART which may cause anxiety.

\section{Limitation of the Study}

The study was cross-sectional; therefore temporal relationship between risk factors and anxiety cannot determined

\section{Conclusion}

Prevalence of anxiety was found to be high on people living with HIV at Debretabor general hospital ART clinic

Being female, perceived stigma started ART and divorced were significantly associated to anxiety.

\section{Recommendation}

- To Debretabor general hospital

- It is good if regular screening of anxiety is considered especially for females among HIV positive patients

- To Amhara regional health burea

- It is better if psychiatric service is given at the hospital

- To Federal ministry of health

- It is good if guidelines are integrated for early detection of anxiety among HIV positive patient

- It will be better if anxiety based training is given for health professionals who are working at ART clinic

- To researchers

- It will be good if more prospective studies are done in the area to explore the temporary relationship of anxiety and associated factors.

\section{Acronyms}
ART
PLWHA
Anti Retro Viral Therapy
People Living With HIV/AIDS

\section{References}

[1] American Psychiatric Association. Diagnostic and statistical manual of mental disorders: DSM-IV-TR®: American Psychiatric Pub; 2000.

[2] Morrison S, Banushi VH, Sarnquist C, Gashi VH, Osterberg L, Maldonado Y, et al. Levels of self-reported depression and anxiety among HIV-positive patients in Albania: a cross-sectional study.Croatian medical journal. 2011; 52(5):622-8.

[3] UNAIDS report. 2008.

[4] UNAIDS. Epidemiological fact sheet on HIV and AIDS. 2009.

[5] WHO. HIV/AIDS and mental health Report by the Secretariat. 20 November 2008.

[6] Ethiopia FDRO, Health M. national mental health strateg. 2012/13 - 2015/16. 
[7] Kemppainen J, Holzemer W, Nokes K, Eller LS, Corless IB, Bunch EH, et al. Self-care management of anxiety and fear in HIV disease. Journal of the Association of Nurses in AIDS Care. 2003; 14(2):21-9.

[8] Enbal Shacham JM, Nur F. Önen, Toshibumi Taniguchi and Edgar Turner Overton. Screening Anxiety in the HIV Clinic. 2012; 16(8):2407-13

[9] Bethesda. Prevalence of positive screen for anxiety and depression in HIV_1infected women across Western Europe and Canada the cranium study. 2012.

[10] B.M. Celesia LN, M.R. Pinzone, C. Coco, R. La Rosa, F. Bisicchia, S. Mavilla, M. Gussio, G. Pellicanò, V. Milioni, F. Palermo, R. Russo, M.T. Mughini, F. Martellotta, R. Taibi, B. Cacopardo, G. Nunnari. High prevalence of undiagnosed anxiety symptoms among HIV-positive individuals on cART: a cross-sectional study. Eur Rev Med Pharmacol Sci 2013;17 (15): 2040-6

[11] M.J. Ho RS, A. Chua, W.M. Chew. Cross-sectional survey of anxiety and depression among HIV Patients at the Communicable Disease Centre (CDC) in Singapore. 2011:17-20.

[12] L. Nogueira Camposa PDFBMDCG. Anxiety and depression assessment prior to initiating antiretroviral treatment in Brazil. 2006 18(6):529-36.

[13] Lorenza Nogueira Campos MDCG, Robert H. Remien Anxiety and Depression Symptoms as Risk Factors for Non-adherence to Antiretroviral Therapy in Brazil 2010; 14(2): 289-99.

[14] Li Liu RP, Wei Sun, Ming Wu, Peng Qu, Chunming Lu and Lie Wang Functional social support, psychological capital, and depressive and anxiety symptoms among people living with HIV/AIDS employed full-time. Department of Social Medicine, School of Public Health, China Medical University, No 92 North 2nd Road, Heping District, Shenyang, Liaoning 110001 , People's Republic of China. 2013; 13(324 ).
[15] Reuters T. Depression and anxiety among HIV-positive sex workers in Kolkata, India: Testing and modifying the Hospital Anxiety Depression Scale. 2013; 56(6).

[16] Namita N. Deshmukh JSD, Avinash M. Borkar and Mohan B. Khamgaonkar. Depression in People living with HIV/AIDS: A Prevalence study inAnti-Retroviral Therapy Centre. Academia and Industrial Research2013; 2(5).

[17] Chandra PS, Ravi V, Desai A, Subbakrishna D. Anxiety and depression among HIV-infected heterosexuals-a report from India. Journal of Psychosomatic Research. 1998; 45(5):401-9.

[18] D Sulyman O, AD Yussuf. Psychiatric Disorders Among People Living With HIV/AIDS Attending Outpatient Clinics At University Of Ilorin Teaching Hospital, Nigeria2012;19(2).

[19] Ogundipe AOJAAEO. Factors associated with anxiety disorders among HIV positive attends of an HIV clinic in Lagos,Nigeria. International journal of STD\&AIDSstd. 2012; 23(6):389_93.

[20] Freeman M, Nkomo N, Kafaar Z, Kelly K. Factors associated with prevalence of mental disorder in people living with HIV/AIDS in South Africa. AIDS care. 2007; 19(10):1201-9.

[21] Kaaya KF. Prevalence of depression and anxiety disorders in HIV positive outpatients in rural Tanzania. AIDS Care Psychological and Socio-medical Aspects of AIDS/HIV2010; 22(4):415-9.

[22] Ngssanga PW. Prevalence of anxiety and depression among HIV/AIDS patients attending the comprehensive Care Centre (CCC), Kenyatta National Hospital (KNH. 2013; 20(5).

[23] Dalgard O, Dowrick C, Lehtinen V, Vazquez-Barquero JL, Casey P, Wilkinson G, et al. Negative life events, social support and gender difference in depression. Social psychiatry and psychiatric epidemiology. 2006; 41(6):444-51. 